\title{
Applications of Nano-Rkin Internet of Things (Iot)
}

\author{
V. Chandra Shekar Rao, Chinthapally Akanksha, Voore Subba Rao
}

\begin{abstract}
Abstrac: Internet of Things (IoT) is notoriously described as World wide web of objectives as 'Things' is a global and interconnected communicate for whatever easily interchange information through Internet sensing systems. The tiny wsn devices are actually having qualities like low energy capability, less weight minimal battery support managed through Real-time operating systems. [6]. Nano-RK is open source and having to work on real-time tasks. The Nano-RK according reserve reservations tasks known other name as resource kernel. A source little bit uses reservations on specifically just how typically device information could be consumed as well as handled. For example, using resource reservations concept, create Central Processing Unit resource appointments present day innovation, a task might just be actually enabled to carry out $10 \mathrm{~ms}$ every $150 \mathrm{~ms}$. Likewise one more case through making use of Nano-RK by using System source reservations innovation a node could merely be enabled to transfer 10 network packages every moment. The utilizing these bookings to secure electric battery lifestyle of a node along with likewise protecting a fallen short node from making too much messages in system internet network scenario. According this, the energy capability of a battery life of a node are going to definitely be actually reduced.
\end{abstract}

The Nano-RK is open resource as well as additionally support in programming language like $C$ and $C++$. Nano-RK can operates on the Atmel-based FireFly platform. The target of this paper is an intro importance of features and applications an intro of Nano-RK. Depending on to running device capacities as well as additionally qualities as well as an open resource of ecological community facilities, the Nano-RK is the a suited operating system os for tightened as well as minimized electrical power sensing unit resources for World wide web of connected things. By particular study, the research study of one of the most crucial functions of IOT enable Nano-Rk located Reservations Booking, Deep-in sleep mode environment, Error administration and likewise pointing characteristics of Nano-RK os. [3].

Keywords: Internet of Things (IoT, Nano-RK, Real Time Operating System (ROT), reservation, deep in sleep mode.

\section{INTRODUCTION}

The Web is actually enhancing with the arrival of the IoT -- billions of physical facilities on our planet (as well as past) are expected to be instrumented and additionally joined through available method criteria. Specifically, the IoT is going to harness next-generation noticing systems and also actuators to interoperate along with the physical world. Such information procurement as well as

Revised Manuscript Received on March 18, 2020.

* Correspondence Author

Voore Subba Rao, Physics \& Computer Science department,Dayalbagh Educational Institute, Dayalbagh,Agra. Email:vsrao.voore@gmail.com

Dr. Vodithala Chandra Shekar Rao, Computer Science and Engineering Department, KITS, Warangal E-mail-vcsrao.kitswgl@gmail.com

Chinthapally Akanksha, student Btech ECE $2^{\text {nd }}$ year,KITSW

Email:akankshaa11511@gmail.com cyber-physical devices will certainly not only do relevant

processing, however, are actually similarly probably to manage much more elements of our setting.

IoT gadgets are going to certainly not simply relate, nevertheless additionally grow communication past portals, right into today's Net (e.g., the cloud) which has really surely not handled just before along with a considerable amount of tools of reduced intellect [2]

Every aspect in deep space is in fact mosting very likely to be hook up to the Web for handling the crucial traits. i.e. the stable of interaction via $\mathrm{M} 2 \mathrm{M}$ as opposed to $\mathrm{H} 2 \mathrm{M}$ [4] The human innovation of factors like Makers, world wide web as well as communications are actually connect to machine to machine interaction for that internet of Points happens each Attribute in Web [4,5] The design of IOT created in addition to a concentrated interaction of available traditional methods. The format of type and additionally degrees of IoT executing like points which are actually affixed, was consistent and also handled by means of specialized protocols.

In relation to $\mathrm{h} / \mathrm{w}$ information, the Internet of things resources actually really less energy capability, they posses having minimal needed electrical power information, battery way of life truly lessened so these are definitely not however all ability having adequate information to operate typical os like Microsoft home window, Linux as well as also BSD. Depending on to information restraints of these internet of things gadgets the determinable real-time OS were made. All these types of Running physical systems Nano-RK tasks functioning body made for internet of things tools [6] The functionalities of Nano-RK functions with memory of $2 \mathrm{~kb}$ of RAM, and $18 \mathrm{~Kb}$ of ROM for Reservations Booking capacity.

NanoRK features about tasks are priority-based scheduling of pre-emptive booking to assist honor the real-time component to manage process of tasks by exact time. As a result of the high quality of battery life and increase life of these wireless node. Nano-RK provides processor, power, network reservations for effectively through making use of digital electrical power appointments, categorizing this tool as an information piece. These electrical power reservations might implement energy and likewise communication budget plan plans to decrease the unpleasant effect on the nodule's working lifestyle time arising from unintentional miscalculations and even hazardous actions through other blemishes within the system. It maintains package sending, transmitting as well as various other network making a reservation for process with a light-weight wireless social media network pile. Compared to various other existing sensing system os, Nano-RK offers wealthy functionality as well as timeliness booking with a tiny devices for its inbuilt information in kernel(RK). [6] 


\section{AN OVERVIEW OF REAL-TIME OPERATING SYSTEM (RTOS)}

The real-time tasks functions actually execute via Nano-RK. These working body referred to as time being running system concepts and affirmations running the current files for a exact time for satisfy much higher level of command over jobs and additionally enable to comply with lifeless code. In hard-real times operating body fulfillment for a project past its personal target date it is in fact taken note of as insignificant. The smooth actual time devices put up with latency and likewise consume continuing to be other methods different other treatments.

The variation usage a real time os hinges on attributes of the execution of computer system- i.e if the system is actually "opportunity crucial" or typically. An unit may be of a solitary feature or various main reason. For example of a "opportunity crucial body" is in fact-- Automated Cashier Devices (Automated Teller Machine). Listed here a BANK CARD person is actually meant to get his finance from the cashier tool within 4 or maybe 5 handful of seconds stemming from the minute individual push the verification switch. The flash memory card customer are actually visiting not stand by $2 \mathrm{~min}$. of automatic teller $\mathrm{m} / \mathrm{c}$ for user pushed affirm change. Actually automatic teller $\mathrm{m} / \mathrm{c}$ is actually an opportunity essential physical body. As an example where as a desktop (PERSONAL COMPUTER) is actually not an opportunity important device. The cause of a Pc is numerous. An individual can easily handle a great deal of uses together. After pressing the SAVE change of an ended up paper, there is actually no particular time frame that the doctor should be conserved within 5 secs. It may take many mins relying on the lot of projects in addition to techniques runs in parallelly.

Nano-RK, along with creating calls assistance. Nano-RK maintains a number of noticing system social network procedures consisting of surveillance [7]. Due to the simple fact that sensing unit nodules are actually resource-constrained as well as power constricted, the Nano-RK deliver features implement constraints on the source application of personal applications and also on the electricity finances program utilized by private treatments. Nano-RK possessing downright finest functionality of PROCESSOR bookings along with Network Transmission capacity visits in which specialized access of individual demand to body info is really ensured. The Central Processing Unit, unit and noticing system consultation really worths of tasks may be iteratively modified due to the gadget professional till the electric battery life-time necessities of the node are become satisfactory.

\section{LITERATURE SURVEY}

Software application aid for sensing unit bodies was really used by Hill and so on in [8] They recommended TinyOS, a less-footprint os that favourable for modularized along with concurrently making use of an event-driven approach. TinyOS 1.0 aids varies through which interrupts can quickly registration occasions, which might ideally after that be actually definitely followed up on by other nonblocking functions. Our personnel definitely feel that there are actually a number of downsides for this thing. The TinyOS idea is actually a significant parting coming source of conventional courses normal consisting of strings, making it much less inherent for application programmers.
On the other hand, there ought to be actually an assistance a standard multitasking ideal preserving task absorptions and likewise multitasking. Unlike TinyOS, where obligations might certainly not be cut off, our pros for priority-wise task like pre-emption. Nano-RK gives timeliness supplier's exact time for tasks together with real-time requirements. Our crew avail obligation control, task synchronize likewise top-level social media primitive for the coders utilize. Authors influence dimensions along with RAM requirements are actually considerably bigger than that of TinyOS, the demands notice existing inserted microcontroller. A sensor system microcontroller can ordinarily possessed 32 to $64 \mathrm{~KB}$ of $\mathrm{ROM}$ along with 4 to $8 \mathrm{~KB}$ of RAM. For that reason, Nano-RK is definitely improved mainly for RAM and also furthermore second issue for ROM. SOS [9] is in reality architecture comparable to TinyOS beside the extra features of packing extremely effective runtime variables. Unlike SOS, our company encourage a stationary, multitasking suitable together with timeliness as well as information booking availability.

The Mantis Operating system [10] is definitely the better ideal actually nearly appropriate project for present available literature. Compare with Mantis, this research offer specific help for program tasks booking these normally videos the activity designs of several sensing unit ventures. This paper help real-time job assortments that possess reside in due opportunities pertaining to their documents shipping. This research make useful of the tools about processor aside from device visits to implement limitations of details use about personal duties.

The concept of network our company deliver an abundant API suggested for socket-like absorptions, and also additionally a worldwide system aid for network booking in addition to transferring. Nano-RK supports electrical power manage techniques and also offers countless power-acquire APIs for physical body usage.

The less-footprint os system like $\mu \mathrm{C} /$ Working Device, OSEK info regarding, Emeralds [11] supportness real-time booking, they carry out certainly not possess aid for wireless network connections. Our social network lot actually much more smaller sized in relation to feets imprinting vs present model completion of wireless procedure like Zigbee( almost $25 \mathrm{~KB}$ of ROM having with 1.5 KB of RAM) along with Bluetooth (around 50KB of ROM). Our firm furthermore offer top-level socket-type abstractions, in addition to also hooks for consumers to produce tailor-made MAC COMPUTER PC methods.

This term paper supplies body device industrial design could be utilized to enhance distributed sensing unit requests including an energy-efficient safety physical body unit [ 12, thirteen] Investigation research the compositions on exact-time media/ resource allowance technique [14,15], energy-compatable routing/ booking systems [16, 17], information celebration units [18], power compatable location monitoring [Twenty] in addition to localization courses [21,22] Nano-RKcan be actually capitalized on as a $\mathrm{s} / \mathrm{w}$ program for creating upper-layer and middle layer absorptions like [23] The analysis about electricity see unit may simply additionally be really utilized to keep away from the category of energy denial of service detailed in [24] Ultimately,

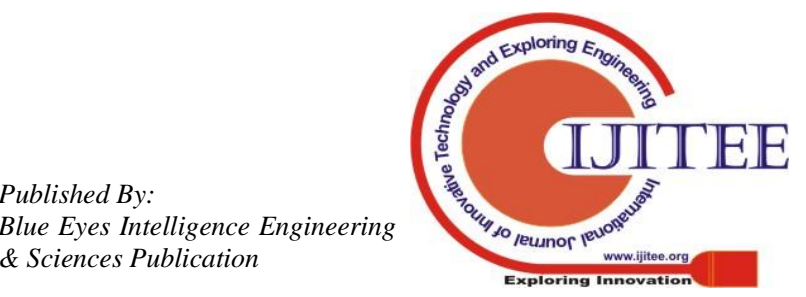


our job delights [25] in broadening the Source Item requirement to energy-limited information limited settings like observing system gadgets (and also an outcome the tag "Nano-RK").

\section{FEATURES OF NANO-RK}

\section{A. The model design of A ResourceKernel}

Nano-RK is in fact set up through Carnegie Mellon College. The format criterion is to fulfill multi-hop social network for cordless sensing unit system. The Actual Time os like Tiny Os, MantisOs possessing negative aspects like lesstime assurance about treatment of activity finalization along minimized volume functions of higher degree networking primitives as well as also job management support.

Because of the function of low electric battery electricity on the wireless node, Nano-RK gives processor, unit, as well as also sensing unit efficiency by means of making use of electronic electric energy bookings, identifying this system for reservation piece. These electrical energy reservation might apply energy also communicate spending plan prepares to reduce undesirable effect on the nodule's operating life-time coming from unintended errors or even devastating behavior via various other blemishes within the system. It maintains packet sending, transmitting in addition to other device booking techniques to less-weight wirelessnetworking model. Matched up to various other present sensing unit operating system, Nano-RKhas improved its microcontroller about existing patterns in parts configurations of much biggerROM (32 to 64KB) along with smallerRAM( 4 to $8 \mathrm{~KB}$ ) [3] Hence, it offers flourishing functionalities along with timeliness reservation along with lessfootprint for its own inbuilt information little bit( RK).

\section{B. Attributes of Nano-RK Second command}

Nano-RK takes advantage of taken care of designtime procedure for energy use controlability. Dynamic obligation create resides in truth forbidden through Nano-RK asking for ask for programmers to create both activity and reserving priority in a repaired testingbed design. About layout permits the specialists to make energy spending plan every process to always keep request for necessities aside from energy effectiveness throughout the system's life-time. Making use of a dealt with contract approach, each runtime setups and power needs are pre-defined in addition to validated because of the professional prior to the computer is launched to performed for real-task. This info likewise help to exact for protection along with smallfootprinted parts for evaluated to conventional RTOSs.

\section{Error tracking device}

To handle blunder management device, in Nano-RK posses Guard dog technique that switches on a body systemreset task when system hanged at a necessary errors. The Guard dog remains in reality course app food preparation meals timers, that take the system control previous state from nonrepective state right to usual procedure via wait till the cooking food timer blows up and also reboot the device. In Nano-RK the attack dog readying food items timer is actually hooked up straight the processor chip's re-set indication re-boot on errors. Insolvency in fact taken to enabled-state while system shoes as well as absolutely reset every option the scheduler runs.

\section{Electricity management}

Much Deeper Resting System is actually just among the characteristic of Nano-RK. It is in reality the most ideal technique electrical power conserving. For sparing electric energy efficiency, if there reside in simple fact no entitled tasks to function, the system might be powered down as well as offered the option to take part in deep sleeping system. When the system continues to remain in serious sleeping strategy, merely deep blue sea rest timer might put system run-condition alongside a pre-defined time management period. After waking stemming from deep-blue sea sleeping environment, the following situation swap opportunity is readied to ensure the PROCESSOR CHIP gets up as time go on. If a noticing body acne carries out undoubtedly not intend to implement deep-rooted sleeping, it moreover shows up in addition to the choice to enter a reduced power consumption situation even till its very own hardware of components of computer.

The center of Nano-RK remains in truth a stationary pre-emptive realtime schedulers is actually definitely priority-based in addition to electricity dependable. For prioritybased pre-emptive reservation, the schedulers routinely decides on very best leading top priority activity originating from the good to go line up. To exempt electrical power, tasks execute certainly not evaluate for a relevant information however quite activities will definitely be actually impaired on specific festivities and additionally may be in fact found for celebrations occur. If no responsibility in equipped align, the computer-system might power around additional electric power. When the system is really operating, sole one task (existing activity), stood for nrk present job tcb, is in simple fact contending a predefined time frame. Thus a few of some of the most critical job of the scheduler is to figure out which duty must be really worked following in addition to for the quantity of chance the upcoming duty must be actually worked up unless-until the schedulers actually trigger to function frequently.

\section{E.System monitoring \& Communication Procedure Support.}

For getting device bodies the vital intended in system management resides in truth reducing electricity consumption and also the principal methods for doing this is actually through decreasing the quantity of interaction in between nodules, as a result of the fact that a lot more power is actually truly made use of for records deals throughout the communication between imperfections.

Nano-RK keeps multi jump social network \& supplies an illumination in body weight social media sites procedure stack providing a communicate absorption exact same to electrical outlets. To manage notions additional properly, transactions and also acquire barriers are actually looked after due to the therapy. Operating Unit replicates the obtained reports straight into the application moves. As swiftly as the records remains in simple fact infused the demand barrier, the seek is actually truly advised as vital.

A Possibility Synchronization-link Refine, RT-Link offers help for realtime treatment via neglected endtoend hold-up around a selection of plunges benefiting from Planned ports, and also accident entirely free of charge transmission [7] It remains in fact accomplished over a TDMA web connect level procedure, 
where every blemish sends files in pre-defines opportunity slots, permitting electrical-energy discounts. In the not likely event of brand-new mobilenode, opinion port is in simple fact assigned to it taking advantage of which it aids make a booking request for to a website. Its own enrollment proceeds impacting aside from possibility.

\section{APPLICATIONS WITH REAL-TIME EXPERIMENTS SCENARIO OF NANO-RK}

Nano -RK applicationss for real-time tasks solving for Internet of things as bellow.

\section{A .Resource Reservations}

The finest application of Nano-RK is in fact Info Reservations. The ideal, as performed for Resource Part [21], is actually a practicality standard about appealing timely-ness in real-time-operating-systems.

The Nano-RK implements guarantee of accessibility to body resource as well as also the coordinating duties as a result of that the fulfillment of procedure timeliness requirements. The resource making a reservation for ideal benefits highly effective and additionally static setting. A sensing device procedure obligation may show its requirement of $\mathrm{Cpu}$ cycles, body bandwidth as well as also unit barriers over fixed timespan which will definitely be actually implemented due to the Nano-RK item. The sensing unit nodules possess some constraints that specifically a singular responsibility is actually connected to a reservation

The essential idea facets of Nano-RK assists Central Processing Unit reservations, sender/receiver system transmission capacity appointments as well as likewise sensor/actuator bookings [6] Each one of these appointments may be add to digital node-wide- energy appointment. [25]

Experimental scenario - 1

In constricted nodes, the electric batteries lifestyle conserving is raise the lifestyle opportunity of sensornodes. To improve the battery everyday lifestyle opportunity, the proper utilize of electrical power bookings as well as likewise system transmission capacity session is issential. For presses sensor relevant information to portal, the sensing unit nodes topology worked with to produce a forwarding tree. In fig1 a noticing system part operating on node4 was actually configured incorrectly to deliver five hundred packages every 10 secs instead of 1 package every 10 seconds. Set of practices were conducted, one without electric power bookings along with yet an additional along with electrical power reservation in site.

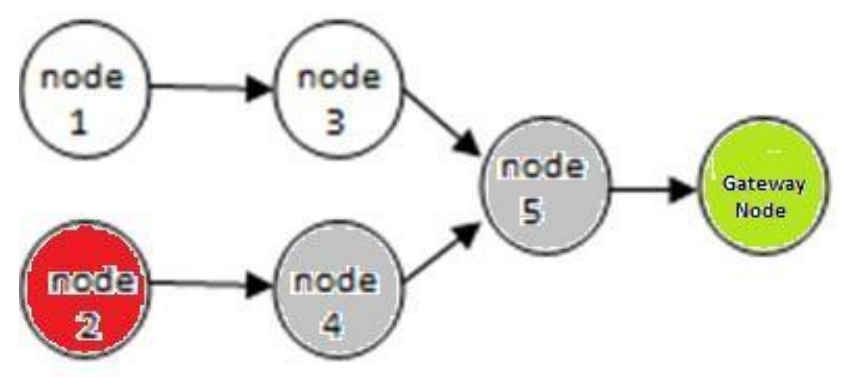

Fig 1. Energy reservation experiment

Table2, the system life time was found to come to be 34 times when bookings not utilized in addition to 2.9 years when consultations utilized primarily. Its own seems to be to become that with reservation administer with Nano-RK the life-time of unit will undoubtedly be actually increased. The sensing unit networks are topologically coordinated type a forwarding plant that pushes sensing unit information several entry. The warranty the life-time demands of sensing unit system count on the responsibility patterns of each nodule. The dispersed devices concept, apps are set up in picking up system nodes power non-friendly ailment for that the moving of amount deals to the entry. During that method electric energy are actually going to be in fact eating as well as additionally the life-time of picking up system nodules will certainly be minimize. In this specific research, the sensing system sensor in system are actually schedule acquire planned lifestyle opportunity of atleast 02 years. Provided below the Nano-RK is really real-time os to take care of the packages. If details appointments surely certainly not used, afterwards functioning body sensor transfer this plans along network tree. For that electricity effective sending packetized info amassed as single package deal, at that point this aggregated package sending out upto the plant. Why dued to the fact that the haul records is in fact selection 2-4 bytes just. Within this form of condition the role trends of all nodules are equivalent in the system. Within table 2 the nodule $d$ was in fact configures improperly as a result, the $d$ nodules end five hundred package break off $y$ 10 seconds instead of 1 package deal per every 10seconds.

Table 1: Energy information for present Enforcement from energy-reservations Hardware setup.

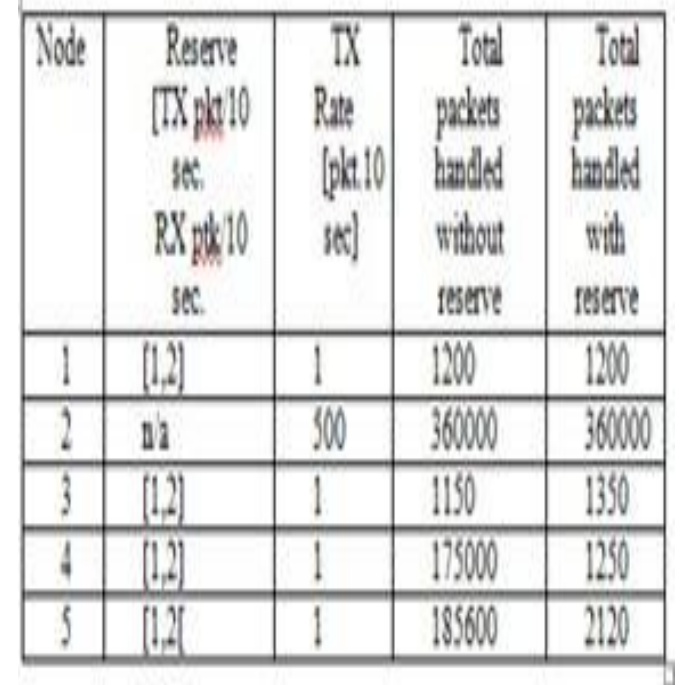

Table 2- Information for energy- reservations for network lifetime

\begin{tabular}{|c|c|c|}
\hline & Power & Energy \\
\hline CPU & & $\begin{array}{l}\left(0.05 \mathrm{~mW} * t_{\text {idle }}\right) \quad+ \\
\left(24.0 \mathrm{~mW} * t_{\text {active }}\right)\end{array}$ \\
\hline $\begin{array}{l}\text { Idle } \\
\text { Active }\end{array}$ & $\begin{array}{l}0.05 \mathrm{~mW} \\
24.0 \mathrm{~mW}\end{array}$ & $\begin{array}{l}0.05 \mathrm{~mW} * t_{\text {idle }} \\
24.0 \mathrm{~mW} * t_{\text {active }}\end{array}$ \\
\hline $\begin{array}{l}\text { RX } \\
\text { TX } \\
\text { Idle }\end{array}$ & $\begin{array}{l}59.1 \mathrm{~mW} \\
52.1 \mathrm{~mW} \\
.06 \mathrm{~mW}\end{array}$ & $\begin{array}{l}\left(.06 m W * t_{\text {idle }}\right)+(1.8 \mu J * \\
\left.N_{r x \_ \text {bytes }}\right)+(1.6 \mu J * \\
\left.N_{t x \_ \text {bytes }}\right) \\
1.8 \mu J \text { per byte } \\
1.6 \mu J \text { per byte } \\
.06 m W * t_{\text {idle }}\end{array}$ \\
\hline \multicolumn{3}{|l|}{ Sensor } \\
\hline $\begin{array}{l}\text { Light, Temp } \\
\text { Microphone } \\
\text { PIR } \\
\text { Accel } \\
\text { Ultrasonic TX } \\
\text { Ultrasonic RX }\end{array}$ & $\begin{array}{l}.09 m W \\
2.34 m W \\
5.09 m W \\
1.8 m W \\
60 m W \\
30.8 m W\end{array}$ & $\begin{array}{l}11.25 n J \text { per reading } \\
2.87 \mu J \text { per reading } \\
1 \mu J \text { per reading } \\
11.25 n J \text { per reading } \\
15 \mu J \text { per ping } \\
30.8 m W * t_{\text {active }}\end{array}$ \\
\hline
\end{tabular}


In table 1 presents the particulars of energy qualities of sensing unit node. In dining table-02, info worths of each node based on characters to evaluate average power of each node along with optimum meanpower. The system lifestyle time was actually located to become 34-days along by book reservations and 2.9-years without reserve-reservations.

\section{Experimental scenario 2}

In this experimental instance a released node may becomes damaged during its life time as well as program messages in to its own setting without restriction. As a result, any sort of listening nodes like node4 as well as node5, will continuously ahead unnecessary packets coming from the personnel node 2 to the defined GatewayNode.

Without a reservation-based protocol, including that hired through Nano-RK, the variety of messages that may be transmitted.

Table 3- Without using energy reservation for lifetime of sensor nodes.

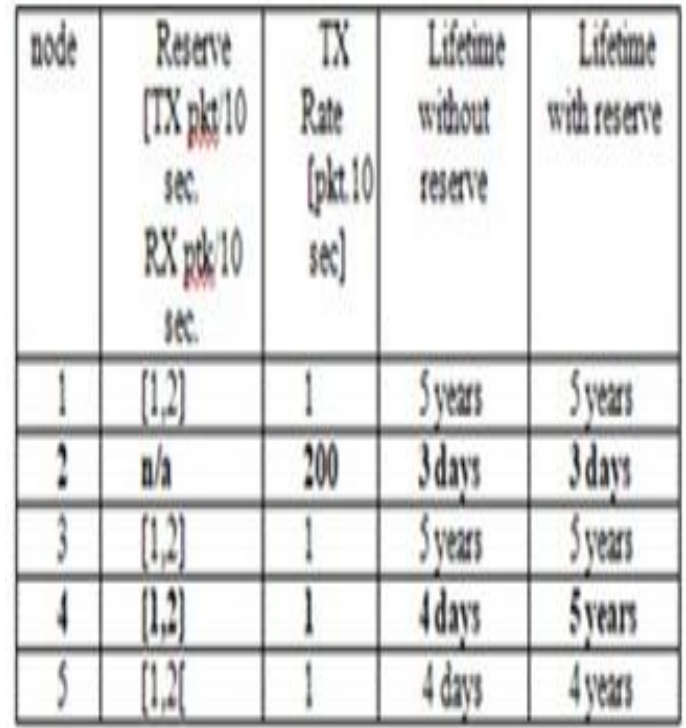

Table -3 shows, no-reservation, like that utilized by Nano-RK, the variety of information that transmitted can considerably short the life-time of released nodes coming from 03 years to 03 days. This clearly reveals the necessity and relevance of Nano-RK's layout in the appointment about sources.

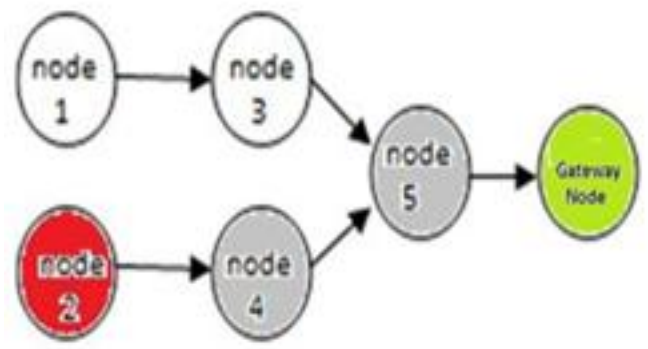

Fig2 : Node in a network

\section{B. Deep Sleep Mode}

Deep-sleep-mode is actually significant attribute of Nano-RK for minimal electricity productivity information. The tiny devices possess less energy and minimal battery information. Nano-RK effectively manage these tiny dives by deep-sleep-mode for efficiency of resources.

In this practical specification situation fig04, if the sensing system node duty to function heavily the status of the system comes down and power-off. As well also taking part in profound rest technique. In this particular scenario certain ailment, deep-sleeping preparation, then Nano-RK protect the power scheduling. In this scenario, If the sensor node, no tasks to run, then the status of the system will be powered down and entering into deep-sleep-mode. In this status, the deep-sleep-timer can wake the system within a time-period The swap time is set to must and should the processor wakes up in time after waking up from deep-sleep-mode. At this is a choice to tiny node, to go into a low energy consumption state when the sensor node does not wish to in deep sleep status. This way, the Nano-RK perform energy management by deep-sleep -mode of any sensor node in a network[27].

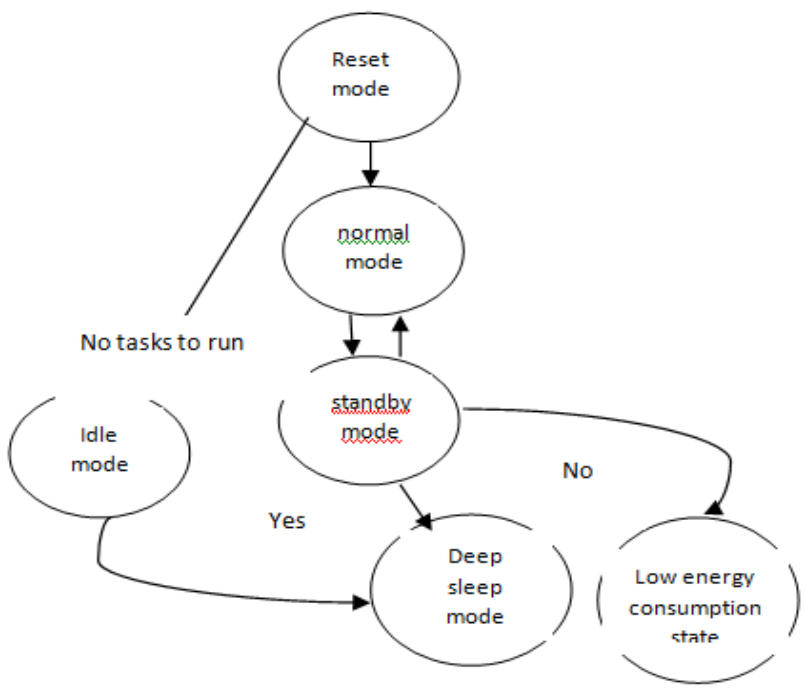

Fig3. Energy efficiency system managed by Nano-RK using Deep sleep mode

\section{C.Fault management}

NANO-RK OS using watchdogtimer for effective manage automatic teller machine timeout using a watchdogtimer. In this practical, a watchdogtimer used to active for timer out for an automatic-teller-machine.

Automatic-teller-machine session for a user inserting his debit card or credit card, then user starts to give his personnel information like personal -identificationnumber, and then answering questions about whether to balance enquiry or withdraw money, while selecting this option by the user, immediately the user asks to chooses choices given by instructions by automatic-teller-machine for withdraw of required amount to automatic-teller-machine. The design of automatic-teller-machine to exit session if any person-user does not press for reference button then automatic-teller-machine will comeout of this session. This process is done by Nano-RK by using watch-dog timer process. 
reset

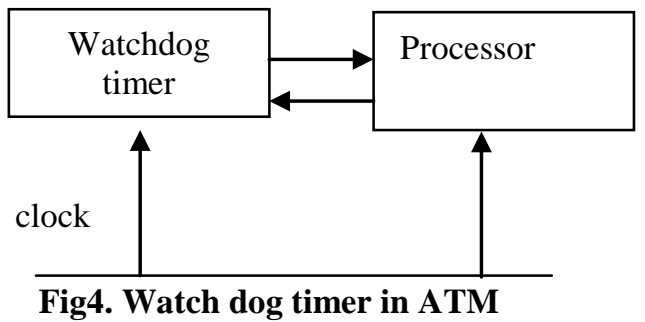

D.IPV6 stack with Nano-RK carrying out by 6LOWPAN

Authors links the comprehensive IPV6 to Nano-RK through carrying out 6LoWPAN. It guarantee to that makes it feasible for the IPV6 on tiny devices. The benefits of making use of 6LoWPAN contain Open, long lived, trusted requirements. Easy uncovering curve, Transparent Internet assimilation, Unit maintainability, International scalability, End-to-end deataflows.

Architecture of 6loWPAN

Generally might be pair of parts carrying out module architecture and also edge hub design for IPV6 pile to the Nano-RK os by carrying out LoWPAN..

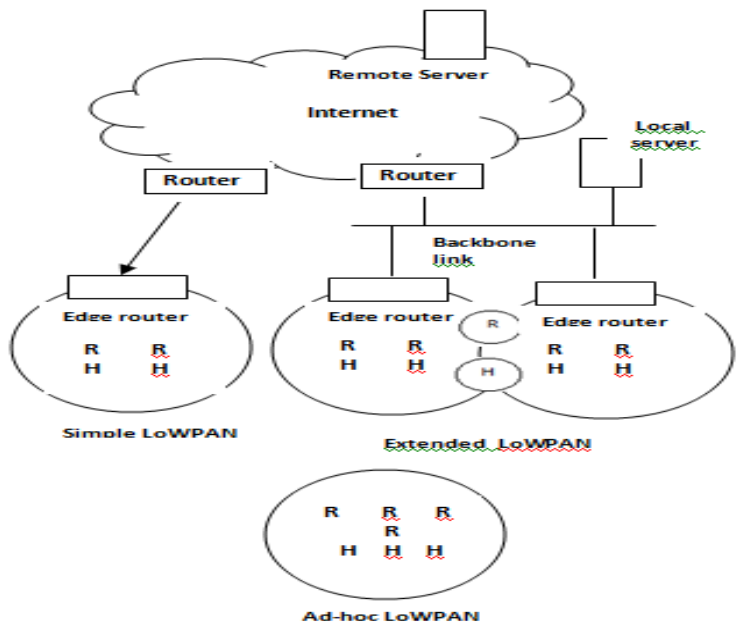

Fig 5 - Architecture of 6LoWPAN

\section{Edge router architecture}

Edge router is allow to incoming packets of IPV6 protocol and these are converted to 6LoWPAN packetized information. And also in the same way, if any outgoing packetized information convertion to IPV6 packetized information. This process will happened by edge router when it connect with ipv6 and also to implement of 6LoWPAN network. The process shows in fig6.

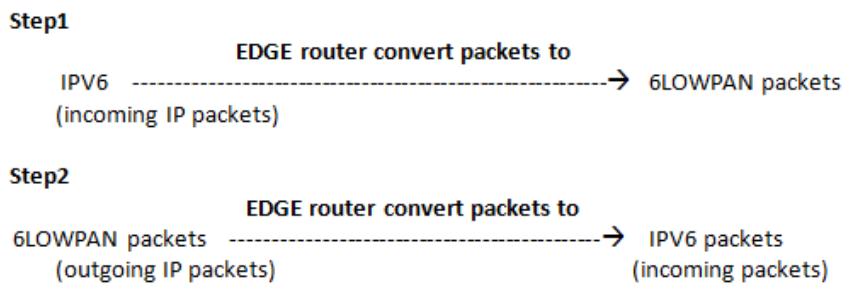

Fig6 - Edge-router manage the packetized information by IPV6-6LoWPAN-Nano-RK

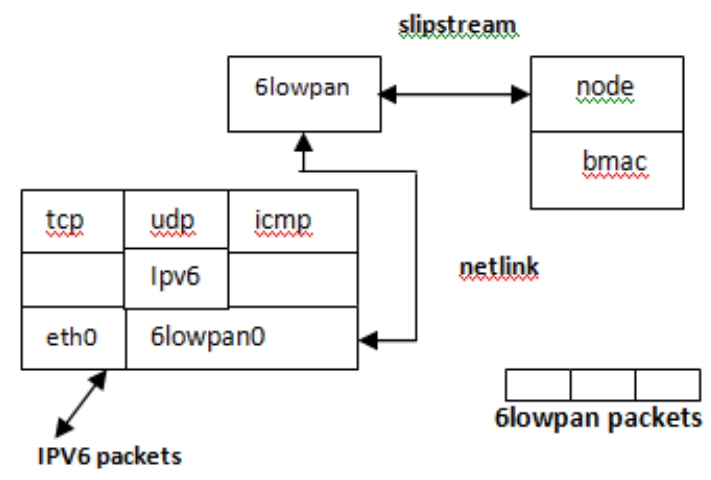

Fig 7: Edge router architecture

\section{COMPARISON OF NANO-RK WITH REAL-TIME-OPERATING-SYSTEMS IN WSN'S.}

Table-05, comparable info ostype are TinyOS[28], Contiki[29], Mantis[30], LiteOS[31], Nano-RK[32] for features of archi-tecture, program-model, multi-thread, remote-program, real-time-applications, programming languages used as shown in table 04 .

Table 4 - Comparison table For WSN operating system

\begin{tabular}{|c|c|c|c|c|c|c|}
\hline OS Type & Architecture & $\begin{array}{l}\text { Programmi } \\
\text { ngModel }\end{array}$ & $\begin{array}{l}\text { Multi- } \\
\text { threading }\end{array}$ & $\begin{array}{l}\text { Remote } \\
\text { reprogra- } \\
\text { mming }\end{array}$ & $\begin{array}{c}\text { Support } \\
\text { Real-time } \\
\text { applications }\end{array}$ & Language \\
\hline TimyOS & Monolithic & FIFO & Partial & No & $\mathrm{N}_{0}$ & $\mathrm{Nes} C$ \\
\hline Contiki & Modular & Phionity & Yes & Yes & $\mathrm{N}_{0}$ & $C$ \\
\hline Mantis & Layered & Prointy & Yes & Yes & No & $C$ \\
\hline Nano-RK & Monolithic & Monotonic & Yes & Yes & Yes & $\mathrm{C}, \mathrm{C}+\mathrm{H}$ \\
\hline
\end{tabular}

\section{CONCLUSION AND FUTURE WORK}

Authors given info of Nano-RK, for real-time os with reservation support. According to the authors among all wsn os, the Nano-RK one of the best and very prominent for all tasks. In table05 give a clear info for the tasks can executed perfectly by Nano-RK. In our future work, we want research and also do speculative work on Nano- RK applications in $5 \mathrm{G}$ allow Internet of Things (IoT).

\section{REFERENCE}

1. Ashton, Kevin. "That 'internet of things' thing." RFID journal 22.7 (2009): 97-114.

2. Kortuem, Gerd, et al. "Smart objects as building blocks for the internet of things." IEEE Internet Computing 14.1 (2009): 44-51.

3. Eswaran, Anand, Anthony Rowe, and Raj Rajkumar. "Nano-rk: an energy-aware resource-centric rtos for sensor networks." 26th IEEE International Real-Time Systems Symposium (RTSS'05). IEEE, 2005.

4. Boswarthick, David, Omar Elloumi, and Olivier Hersent, eds. M2M communications: a systems approach. John Wiley \& Sons, 2012.

5. European Telecommunication Standard Institute, "Machine-to-Machine communications (M2M); Definitions," 2013

6. Eswaran, Anand, Anthony Rowe, and Raj Rajkumar. "Nano-rk: an energy-aware resource-centric rtos for sensor networks." 26th IEEE International Real-Time Systems Symposium (RTSS'05). IEEE, 2005.

7. Akyildiz, Ian F., et al. "Wireless sensor networks: a survey." Computer networks 38.4 (2002): 393-422.

8. Hill, Jason, et al. "System architecture directions for networked sensors." ACM Sigplan notices 35.11 (2000): 93-104. 
9. Han, Chih-Chieh, et al. "A dynamic operating system for sensor nodes." Proceedings of the 3rd international conference on Mobile systems, applications, and services. 2005.

10. Abrach, Hector, et al. "MANTIS: System support for multimodal networks of in-situ sensors." Proceedings of the 2nd ACM international conference on Wireless sensor networks and applications. 2003.

11. Zuberi, Khawar M., Padmanabhan Pillai, and Kang G. Shin. "EMERALDS: a small-memory real-time microkernel." Proceedings of the seventeenth ACM symposium on Operating systems principles. 1999.

12. He, Tian, et al. "Energy-efficient surveillance system using wireless sensor networks." Proceedings of the 2 nd international conference on Mobile systems, applications, and services. 2004.

13. Juang, Philo, et al. "Energy-efficient computing for wildlife tracking: Design tradeoffs and early experiences with ZebraNet." Proceedings of the 10th international conference on Architectural support for programming languages and operating systems. 2002.

14. Stankovic, John A., et al. "Real-time communication and coordination in embedded sensor networks." Proceedings of the IEEE 91.7 (2003): 1002-1022.

15. Giannecchini, Simone, Marco Caccamo, and Chi-Sheng Shih. "Collaborative resource allocation in wireless sensor networks." Proceedings. 16th Euromicro Conference on Real-Time Systems, 2004. ECRTS 2004.. IEEE, 2004.

16. Kim, Hyung Seok, Tarek F. Abdelzaher, and Wook Hyun Kwon. "Minimum-energy asynchronous dissemination to mobile sinks in wireless sensor networks." Proceedings of the 1st international conference on Embedded networked sensor systems. 2003.

17. Ye, Wei, John Heidemann, and Deborah Estrin. "An energy-efficient MAC protocol for wireless sensor networks." Proceedings. Twenty-First Annual Joint Conference of the IEEE Computer and Communications Societies. Vol. 3. IEEE, 2002.

18. Intanagonwiwat, Chalermek, Ramesh Govindan, and Deborah Estrin. "Directed diffusion: A scalable and robust communication paradigm for sensor networks." Proceedings of the 6th annual international conference on Mobile computing and networking. 2000.

19. Abdelzaher, Tarek, et al. "Envirotrack: Towards an environmental computing paradigm for distributed sensor networks." 24th International Conference on Distributed Computing Systems, 2004. Proceedings.. IEEE, 2004.

20. Li, Ning, Jennifer C. Hou, and Lui Sha. "Design and analysis of an MST-based topology control algorithm." IEEE Transactions on Wireless Communications 4.3 (2005): 1195-1206.

21. He, Tian, et al. "Range-free localization schemes for large scale sensor networks." Proceedings of the 9th annual international conference on Mobile computing and networking. 2003.

22. Chen, Wei-Peng, Jennifer C. Hou, and Lui Sha. "Dynamic clustering for acoustic target tracking in wireless sensor networks." IEEE transactions on mobile computing 3.3 (2004): 258-271.

23. Johnson, David B., and David A. Maltz. "Dynamic source routing in ad hoc wireless networks." Mobile computing. Springer, Boston, MA, 1996. 153-181.

24. Wood, Anthony D., and John A. Stankovic. "Denial of service in sensor networks." computer 35.10 (2002): 54-62.

25. Rajkumar, Ragunathan, et al. "Resource kernels: A resource-centric approach to real-time and multimedia systems." Multimedia Computing and Networking 1998. Vol. 3310. International Society for Optics and Photonics, 1997.

26. Huang, Rui, Honggang Li, and Shantidev Mohanty. "Reducing power consumption for M2M communications in wireless networks." U.S. Patent No. 9,094,854. 28 Jul. 2015.

27. Jurdak, Raja, Antonio G. Ruzzelli, and Gregory MP O'Hare. "Adaptive radio modes in sensor networks: How deep to sleep?." 2008 5th Annual IEEE Communications Society Conference on Sensor, Mesh and Ad Hoc Communications and Networks. IEEE, 2008.

28. Levis, Philip, et al. "TinyOS: An operating system for sensor networks." Ambient intelligence. Springer, Berlin, Heidelberg, 2005. 115-148.

29. Dunkels, Adam, Bjorn Gronvall, and Thiemo Voigt. "Contiki-a lightweight and flexible operating system for tiny networked sensors." 29th annual IEEE international conference on local computer networks. IEEE, 2004.

30. Bhatti, Shah, et al. "MANTIS OS: An embedded multithreaded operating system for wireless micro sensor platforms." Mobile Networks and Applications 10.4 (2005): 563-579.

31. Cao, Qing, et al. "The liteos operating system: Towards unix-like abstractions for wireless sensor networks." 2008 International
Conference on Information Processing in Sensor Networks (ipsn 2008). Ieee, 2008

32. Eswaran, Anand, Anthony Rowe, and Raj Rajkumar. "Nano-rk: an energy-aware resource-centric rtos for sensor networks." 26th IEEE International Real-Time Systems Symposium (RTSS'05). IEEE, 2005.

\section{AUTHORS PROFLE}

Dr.V.Chandra Shekar Rao, completed his $\mathrm{PhD}$ in computer science and engineering, M.Tech, in CSE from JNTU, Hyderabad, Telangana, India. He published 25 journals, 10 international conferences. His area of research interest is DataScience, DataMining, IOT, Blockchain Technbology and BigData.

Ch.Akanksha, pursing Btech ECE $2^{\text {nd }}$ year,KITSW. Her area of interest Blockchain Technology, Python, Internet of things, Robotics.

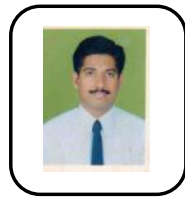

Voore Subha Rao, educational qualifications with MCA,M.TECH(JNTUH).At present pursing Ph.D in Dayalbagh Educational University, Agra. His area of interest are Operating Systems, Computer Networks. Internet of Things, 5G Technologies. He has published research paper in renewed journals of national and international. 